1 Supporting Information

\title{
2 Multigene Pathway Engineering with 3 Regulatory Linkers (M-PERL)
}

4

5
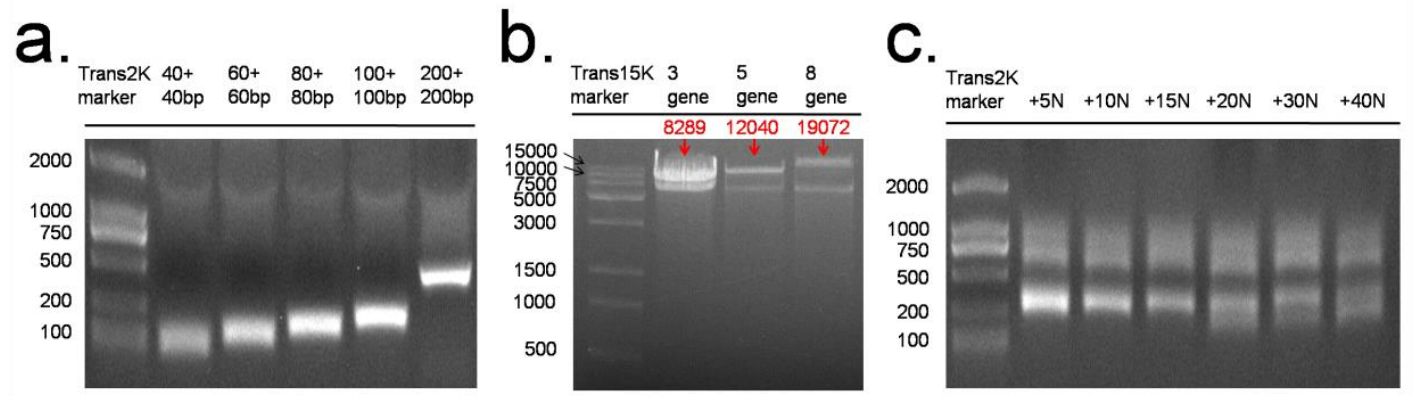

Figure S1. The results of gel electrophoresis. a.) The different DNA linkers joining the cistron 1 and cistron 2 were shown as examples. The linkers contain both homologous ends at different lengths of 40, 60, 80, 100 and 200 bases generating the whole linkers of $80,120,160,200$, and $400 \mathrm{bp}$. b.) The positive results of separate assemblies of 3, 5, and 8 genes on pRS415 vector were verified by Not I digestion. The two Not I sites outside the whole assembled pathway are introduced by linkers. c.) The exampled linkers constructed by T-PCR and F-PCR were incharge of joining the promoter PDC1p and the part $g f p$ 's ORF. The linkers contained $120 \mathrm{bp}$ of the left homologous ends and $150 \mathrm{bp}$ of the right homologous ends and the different lengths of $5,10,15,20,30$, and 40 bases of $\mathrm{N}(\mathrm{A} / \mathrm{T} / \mathrm{C} / \mathrm{G})$. 


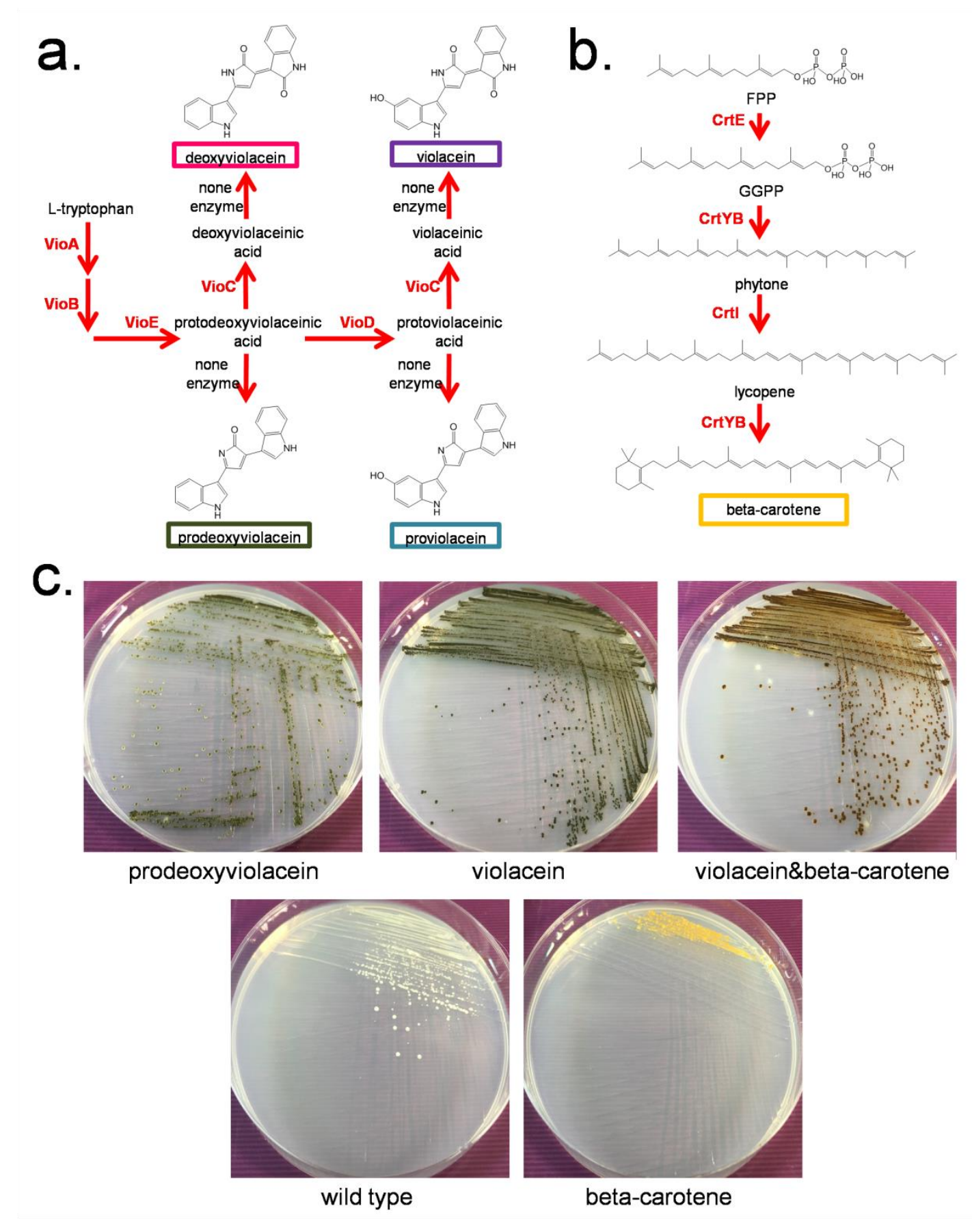

Figure S2. The multigene pathways constructed in this work. The illustrations of constructed pathways are listed. a.) A complete violacein synthesis heterologous pathway consists of five genes as vioA, vioB, vioE, vioD, vioC. With combinations of different reactions charged by the five enzymes, the pathway contains four main branched products as prodeoxyviolacein, deoxyviolacein, proviolacein and violacein. b.) A complete beta-carotene heterologous pathway consists of three genes which govern four reactions from FPP to the final product. c.) The production of different products made the yeast strain present different colors. The prodeoxyviolacein synthesis pathway made the colony green, the violacein synthesis pathway made the colony dark purple or nearly black, and the co-existence of the violacein synthesis 
pathway and the beta-carotene synthesis pathway made the colony dark brown, comparing with the orange color of the colony containing only beta-carotene pathway.
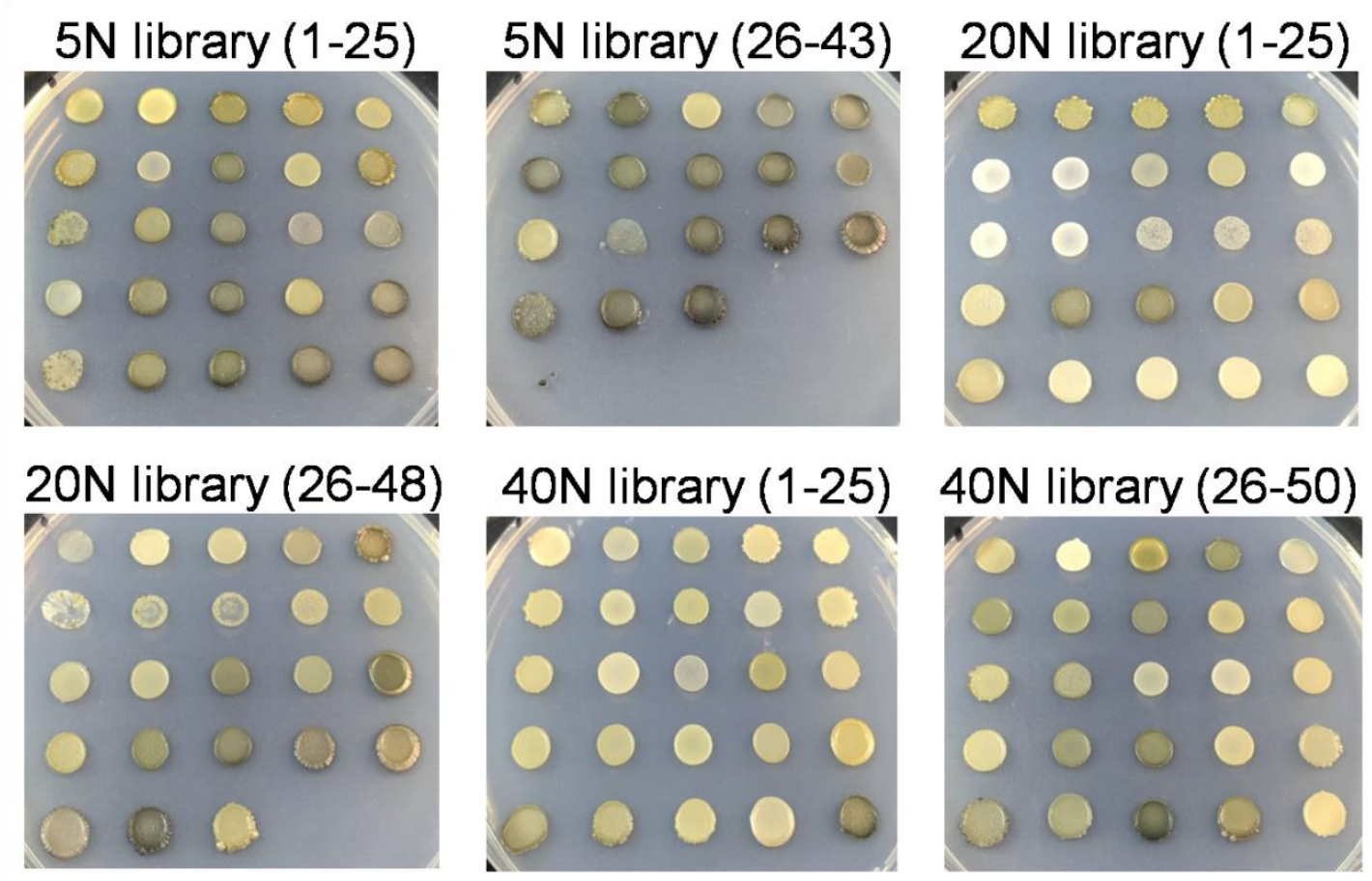

Figure S3. The variety of violacein-synthesis cell libraries generated by $5 \mathrm{~N}, 20 \mathrm{~N}$ and $40 \mathrm{~N}$ linker libraries. The $5 \mathrm{~N}, 20 \mathrm{~N}$ and $40 \mathrm{~N}$ linker libraries meant the linkers containing 5, 20 and 40 random bases of N, which were already explained in Figure 4 and 5. Different linker libraries generated different cell libraries named as $5 \mathrm{~N}, 20 \mathrm{~N}$, and $40 \mathrm{~N}$ libraries. All the colonies spotted on the plates were related to the samples detected in the Figure 5.

$5 \mathrm{~N}-12$

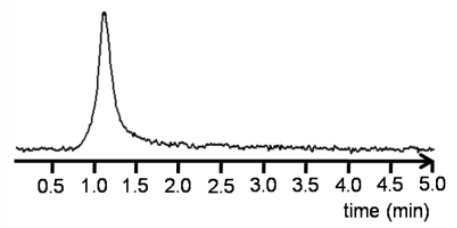

$5 \mathrm{~N}-25$

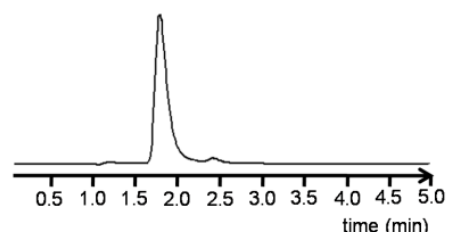

$5 \mathrm{~N}-22$

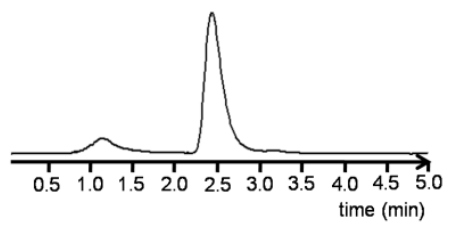

$5 \mathrm{~N}-43$

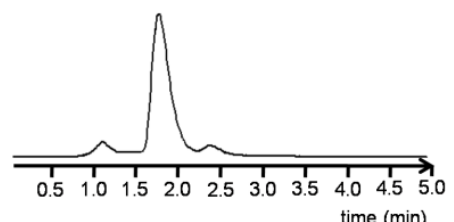

$5 \mathrm{~N}-17$

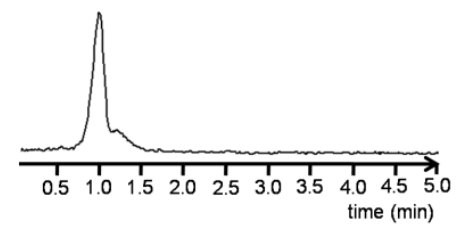

$20 N-48$

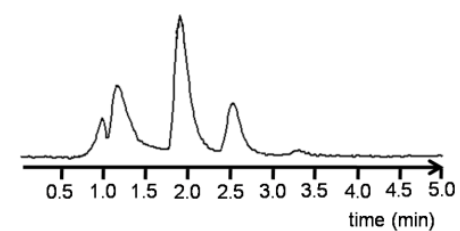

Figure S4. The chromatogram and absorbance spectra of representative samples of cells'liraries for violacein synthesis. Some example strains from the assembled 
libraries showed the varied product compositions. The four main products were detected at their specified elution times and wavelengths $(1.88 \mathrm{~min} / 574 \mathrm{~nm}$ for violacein and $2.42 \mathrm{~min} / 562 \mathrm{~nm}$ for deoxyviolacein; $0.97 \mathrm{~min} / 600 \mathrm{~nm}$ for proviolacein; $1.16 \mathrm{~min} / 610 \mathrm{~nm}$ for prodeoxyviolacein).

a.

Natural promoters

$$
\text { WT }
$$

Artificial promoters

M1 Truncated just from TSS

M2 Truncated after TSS

M3 Truncated by Kozak 4bp

M4 TSR replaced by that of PGI1p

M5 TSR replaced by that of TDH3p

M6 Elongated an extra TSR of PGI1p

M7 Elongated an extra TSR of TDH3p

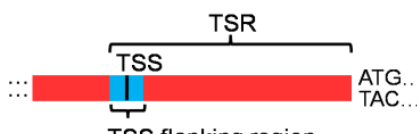

TSS flanking region
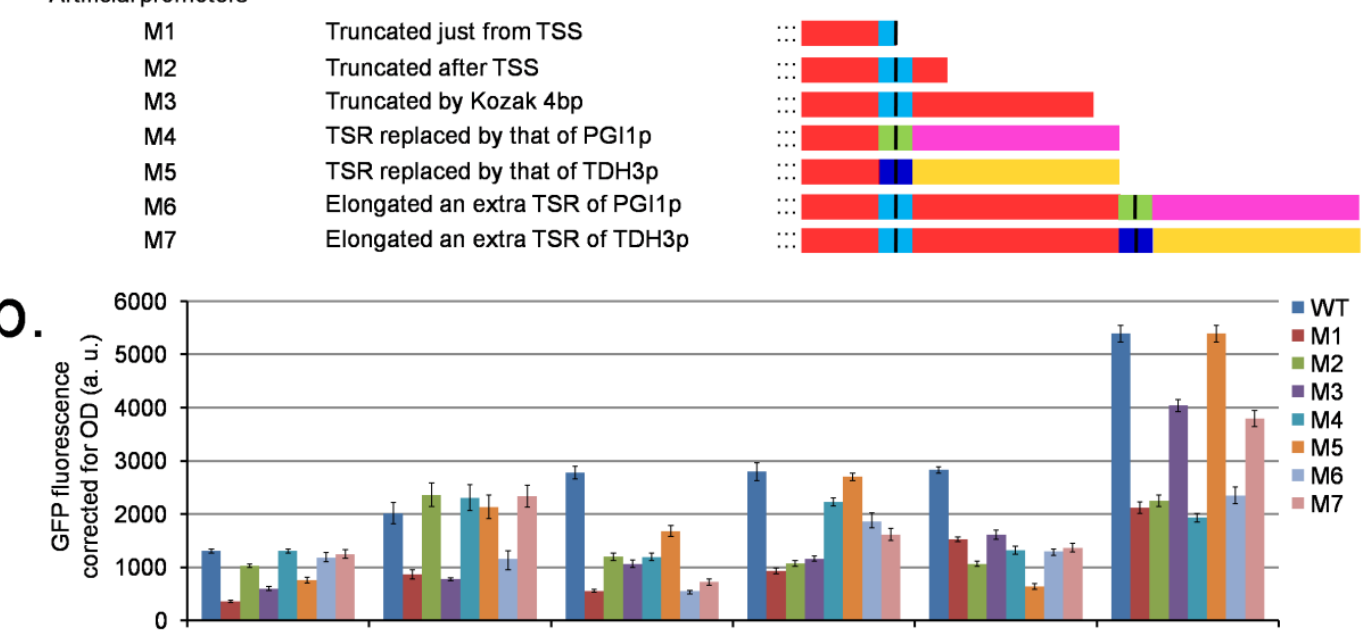

PGI1p

ENO2p

PGK1p

TEF1p

TPI1p

$\mathrm{TDH} 3 \mathrm{p}$

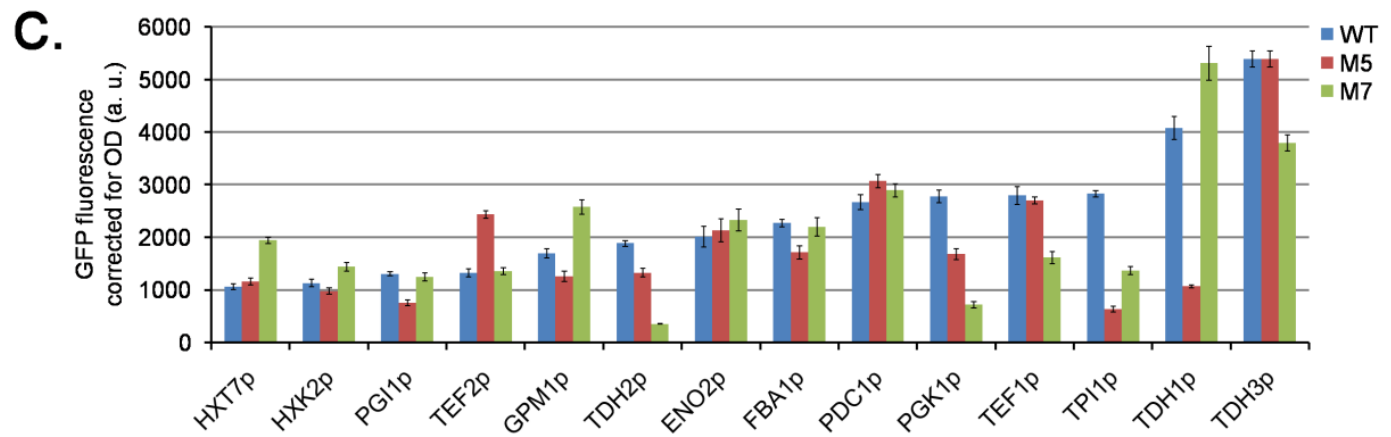

Figure S5. The influences of truncated, replaced and elongated TSS and adjacent

60 regions on promter's strenghs. a.) The special truncated, replaced and elongated TSS and TSR sequence changes were introduced into promoter engineering. TSS: transcriptional start site; TSR: transcriptional start region. b. c.) The impacts of special sequences changes on different promoters' strengths. GFP fluorescence corrected for OD600 was measured. All error bars indicate $\pm \mathrm{SD}, \mathrm{n}=3$. 


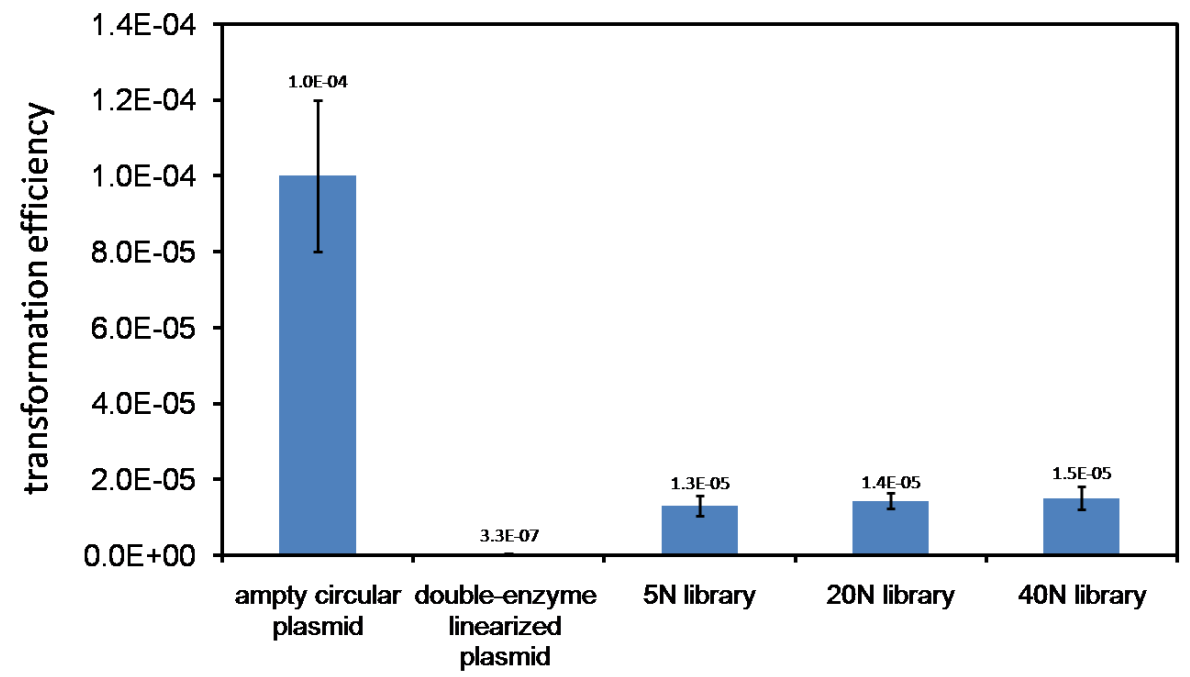

Figure S6. The compared transformation efficiencies of different DNA products. The same moles of different DNA products were separately transformed into yeast cells. Cells were plated and the transformation efficiencies were calculated. The experiments were operated with three replicates.

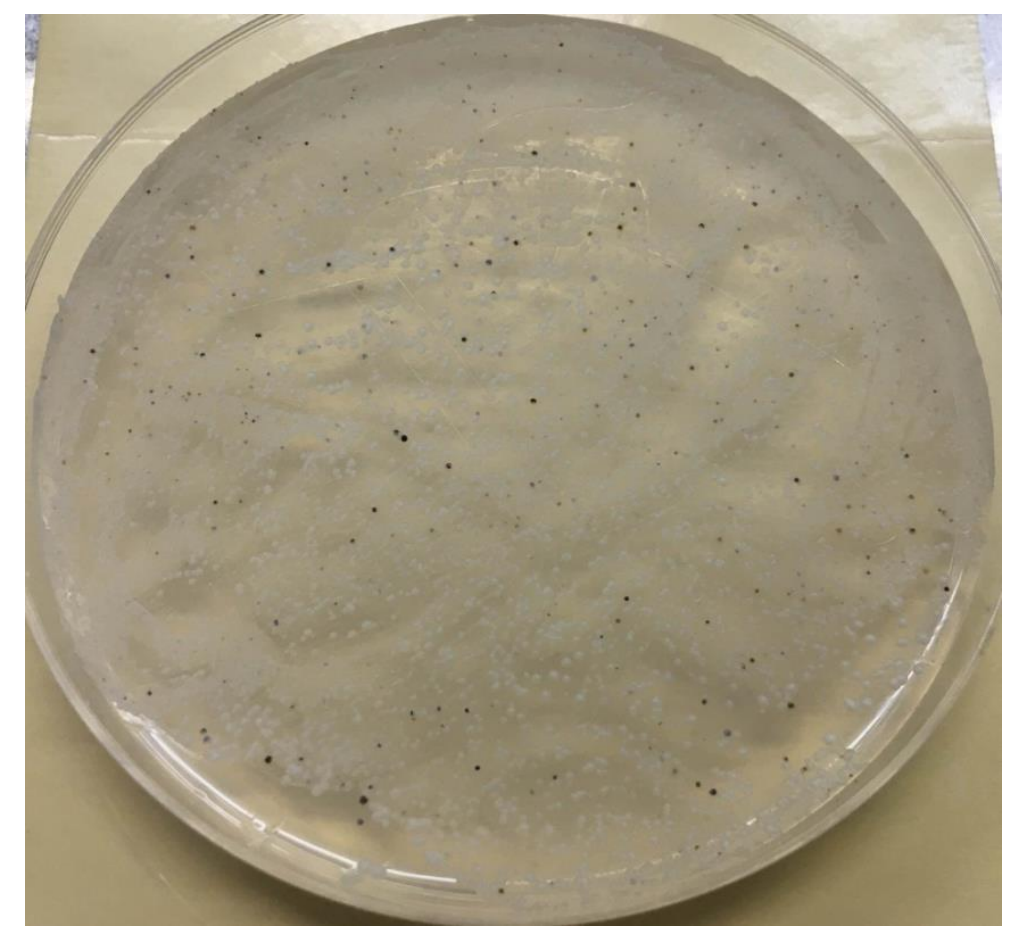

Figure S7. A $20 \mathrm{~N}$ cell library was constructed for the industrial "Angel Yeast". A 20N library was constructed for an alpha type haploid of the industrial "Angel Yeast". All the DNA materials used in 20N library construction for BY4741 were also used here, except for the vector was changed into a Not I-linearized pRS416. The result was shown as the photo of the plate.

The figure S7 showed that a library could also be successfully constructed in an industrial yeast strain by using M-PERL strategy. The use of industrial "Angel Yeast" got higher transformation efficiency $\left(10^{-4}\right)$ but lower assembly efficiency (10-20\%) 
than the use of lab's strain BY4741. The possible reasons lay in some aspects. First, the higher robustness of "Angel yeast" made more cells alive after transformation. Second, the higher efficiency of manipulation made BY4741 get higher percentage of correct results. Third, the vector linearized by double enzymes other than single enzyme might be more beneficial to DNA assembly.

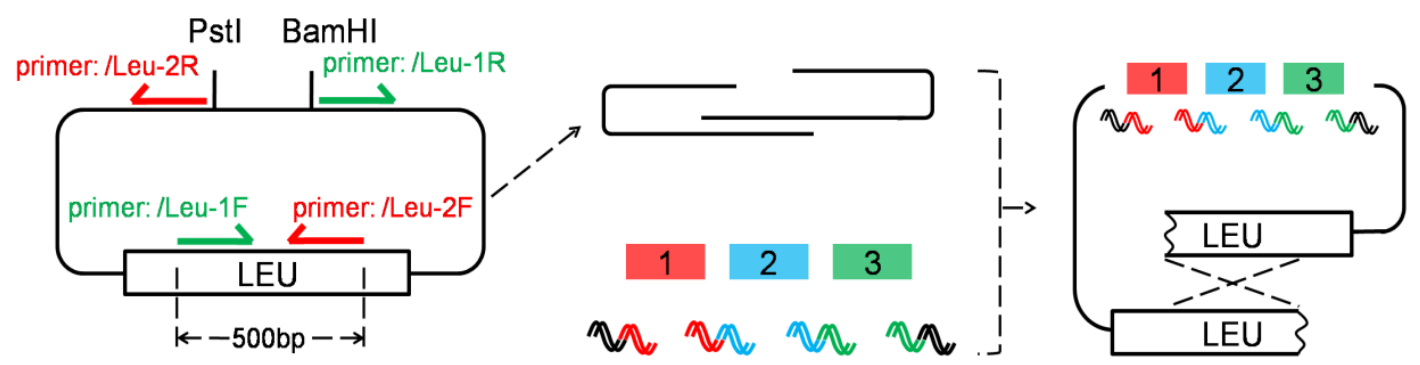
pRS425

Figure S8. Selection of correct assembled results by using the two pieces of multi-copy vector pRS425. A general process was designed to improve the multigene assembly efficiencies on multi-copy vector pRS425. Two sub-groups of primers were utilized in two cloning reactions, and pRS425 was divided into two pieces owning $500 \mathrm{bp}$ homologous ends in the marker's sequence with each other. When the two pieces of the vector were transformed into yeast together with other DNA segments, the cell had to recombine the whole active vector first to offer an integrate platform for the assembly of other DNA parts.

The process in figure S8 was used to test 3, 5 and 8 gene assembly in the main text and the related results were discussed. Here the more detailed causes were discussed. This primary demand of recombining two pieces of the vector directly led to higher selection pressure and decreased numbers of the total living cells after yeast transformation. In another word, the transformation efficiencies were weakened sharply as discussed in the main text. On the other hand, as the living cells became less, the numerators and denominators for calculating assembly efficiencies also turn small (separately into 1-10 and 10-100). As a result, the plan of using "two-piece-vector" resulted in improved assembly efficiencies. It was obvious that the "two-piece-vector" choice was beneficial to selecting correct assembly results but actually detrimental to building cell libraries. Due to the above results, the enzyme-digested vector was superiorly chosen for library construction other than the two-piece vector.

Table S1. The main primers used in this work.As shown in Table S1, most of the oligo names consisted of two parts owning different senses. As an example, for "LP1-G-1", the first "LP1-G" meant "linker between P1 and gfp's ORF", and the second "-1" meant "the first oligo designed for linker synthesis". This group of oligos was used in the single promoter engineering experiments. As another example, for "LTC1-2-4(20N)", the first "LTC1-2" meant "linker between transformed cistron 1 and 2", and the second " $4(20 \mathrm{~N})$ " meant "the 4th oligo designed for linker 
synthesis"and "inserting 20bp of $\mathrm{N}$ as regulatory sequence". This group of oligos was used in violacein pathway library experiments.

\begin{tabular}{|c|c|}
\hline Primer & 5 ' to 3'Sequence \\
\hline /Leu-1F & AGGAATTCGATATCAAGCTTATCGATACCGTCGAC \\
\hline /Leu-1R & CCACTAGTTCTAGAGCGGCCGCCACCGCGGTGGAG \\
\hline /Leu-2F & CCAAACATGTTGCTGGTGATTATAATACCATTTAG \\
\hline /Leu-2R & GGGTACCGGTAGTGTTAGACCTGAACAAGGTTTAC \\
\hline PDC1p-F & TATTTTAGATTCCTGACTTCAACTCAAG \\
\hline PDC1p-R & TGTTTTATATTTGTTGTAAAAAGTAGATAATTAC \\
\hline PGK1p-F & GGTGGGTGTGGGTGTATTG \\
\hline PGK1p-R & TTTTAGGCTGGTATCTTGATTCT \\
\hline PGI1p-F & TATATCTAGGAACCCATCAGGTTG \\
\hline PGI1p-R & TTTTAGTTTATGTATGTGTTTTTTGTAGT \\
\hline TPI1p-F & ACTTCTCGTAGGAACAATTTCG \\
\hline TPI1p-R & TTTTTGATTAAAATTAAAAAAACTTTTTG \\
\hline HXT7p-F & TATTTTAGATTCCTGACTTCAACTCAAG \\
\hline HXT7p-R & TGTTTTATATTTGTTGTAAAAAGTAGATAATTAC \\
\hline TEF1p-F & AATGTTTCTACTCCTTTTTTACTCTTCCAGATTTTC \\
\hline TEF1p-R & TTTGTAATTAAAACTTAGATTAGATTGCTATGC \\
\hline TDH3p-F & ACAGTTTATTCCTGGCATCCACTAAATATAATG \\
\hline TDH3p-R & TTTGTTTGTTTATGTGTGTTTATTCGAAACTAAGTTC \\
\hline FBA1p-F & TGGGTCATTACGTAAATAATGATAG \\
\hline FBA1p-R & TTTGAATATGTATTACTTGGTTATGG \\
\hline TDH1p-F & TGTATATGCTCATTTACACTCTATATCAC \\
\hline TDH1p-R & TTTGTTTTGTGTGTAAATTTAGTGAAG \\
\hline GPM1t-F & GTCTGAAGAATGAATGATTTGATG \\
\hline GPM1t-R & TATTCGAACTGCCCATTCAG \\
\hline GPDt-F & GTGAATTTACTTTAAATCTTGCATT \\
\hline GPDt-R & GGAATCTGTGTATATTACTGCATCTAG \\
\hline FBA1t-F & GTTAATTCAAATTAATTGATATAGTTTTTTAATG \\
\hline FBA1t-R & AAAGATGAGCTAGGCTTTTGTAAAAATATC \\
\hline PGK1t-F & ATTGAATTGAATTGAAATCGATAG \\
\hline PGK1t-R & AACGAACGCAGAATTTTCG \\
\hline CYC1t-F & CATGTAATTAGTTATGTCACGCTTAC \\
\hline CYC1t-R & AAAGCCTTCGAGCGTCCC \\
\hline PDX1t-F & ATAAAAAACACGCTTTTTCAGTTCGAGTTTATC \\
\hline PDX1t-R & TCACCCAGACACCTACGATG \\
\hline MPE1t-F & ATAACAATACTGACAGTACTAAATAATTG \\
\hline MPE1t-R & CACATTTTTGAGATTTTCAACTATTAC \\
\hline TDH2t-F & ATTTAACTCCTTAAGTTACTTTAATGATTTAGT \\
\hline TDH2t-R & GCGAAAAGCCAATTAGTGTGATAC \\
\hline vioA-F & ATGAAACATTCTTCCGATATC \\
\hline vioA-R & TTACGCGGCGATACGCTGCAAC \\
\hline vioB-F & ATGAGCATTCTGGATTTCCCG \\
\hline
\end{tabular}




\begin{tabular}{|c|c|}
\hline vioB-R & TTAGGCCTCGCGGCTCAGTTTG \\
\hline vioE-F & ATGGAGAACCGTGAGCCACC \\
\hline vioE-R & TTAGCGCTTGGCCGCGAAAAC \\
\hline vioD-F & ATGAAGATTCTGGTCATTGG \\
\hline vioD-R & TTATCAGCGCTGCAAAGCAT \\
\hline vioC-F & ATGAAACGTGCGATTATCG \\
\hline vioC-R & TTAATTCACGCGACCAATCTTGTACC \\
\hline crtE-F & ATGGATTACGCGAACATCCTC \\
\hline crtE-R & TCACAGAGGGATATCGGCAAG \\
\hline crtI-F & ATGGGAAAAGAACAAGATCAGG \\
\hline crtI-R & TCAGAAAGCAAGAACACCAACG \\
\hline crtYB-F & ATGACGGCTCTCGCATATTACC \\
\hline crtYB-R & TTACTGCCCTTCCCATCCG \\
\hline gfp-F & ATGTCTAAAGGTGAAGAATTATTC \\
\hline gfp-R & TTATTTGTACAATTCATCCATACC \\
\hline LP1-G-1 & $\begin{array}{l}\text { СTTTCACTCTCCTTGCAATCAGATTTGGGTTTGTTCCCTTTATTTTCATA } \\
\text { TTTCTTGTCATATTC }\end{array}$ \\
\hline LP1-G-2 & $\begin{array}{l}\text { GAGGTTATGAGTAGAAAATAATAATTGAGAAAGGAATATGACAAGAAA } \\
\text { TATGAAAATAAAGGGAA }\end{array}$ \\
\hline LP1-G-3 & $\begin{array}{l}\text { TTTCTCAATTATTATTTTCTACTCATAACCTCACGCAAAATAACACAGTC } \\
\text { AAATCAATC }\end{array}$ \\
\hline LP1-G-4(5N) & $\begin{array}{l}\text { ACCAGTGAATAATTCTTCACCTTTAGACATTTTGNNNNNTTTGATTGAT } \\
\text { TTGACTGTGTTATTTTGCG }\end{array}$ \\
\hline LP1-G-4(10N) & $\begin{array}{l}\text { ACCAGTGAATAATTCTTCACCTTTAGACATTTTGNNNNNNNNNNTTTG } \\
\text { ATTGATTTGACTGTGTTATTTTGCG }\end{array}$ \\
\hline LP1-G-4(15N) & $\begin{array}{l}\text { ACCAGTGAATAATTCTTCACCTTTAGACATTTTGNNNNNNNNNNNNNN } \\
\text { NTTTGATTGATTTGACTGTGTTATTTTGCG }\end{array}$ \\
\hline LP1-G-4(20N) & $\begin{array}{l}\text { ACCAGTGAATAATTCTTCACCTTTAGACATTTTGNNNNNNNNNNNNNN } \\
\text { NNNNNNTTTGATTGATTTGACTGTGTTATTTTGCG }\end{array}$ \\
\hline LP1-G-4(30N) & $\begin{array}{l}\text { ACCAGTGAATAATTCTTCACCTTTAGACATTTTGNNNNNNNNNNNNNN } \\
\text { NNNNNNNNNNNNNNNNTTTGATTGATTTGACTGTGTTATTTTGCG }\end{array}$ \\
\hline LP1-G-4(40N) & $\begin{array}{l}\text { ACCAGTGAATAATTCTTCACCTTTAGACATTTTGNNNNNNNNNNNNNN } \\
\text { NNNNNNNNNNNNNNNNNNNNNNNNNNTTTGATTGATTTGACTGTGTT } \\
\text { ATTTTGCG }\end{array}$ \\
\hline LP1-G-5 & $\begin{array}{l}\text { CTAAAGGTGAAGAATTATTCACTGGTGTTGTCCCAATTTTGGTTGAATT } \\
\text { AGATGGTGATG }\end{array}$ \\
\hline LP1-G-6 & $\begin{array}{l}\text { CCGGAGACAGAAAATTTGTGACCATTAACATCACCATCTAATTCAACC } \\
\text { AAAATTG }\end{array}$ \\
\hline LP1-G-7 & $\begin{array}{l}\text { GTCACAAATTTTCTGTCTCCGGTGAAGGTGAAGGTGATGCTACTTACG } \\
\text { GTAAATTGACC }\end{array}$ \\
\hline LP1-G-8 & AGTAGTACAAATAAATTTTAAGGTCAATTTACCGTAAGTAGCATCA \\
\hline LP2-G-1 & GCATAAATTGGTCAATGCAAGAAATACATATTTGGTCTTTTCTAATTCGT \\
\hline
\end{tabular}




\begin{tabular}{|c|c|}
\hline & AGTTTTTCAAG \\
\hline LP2-G-2 & $\begin{array}{l}\text { GATCTGTAAAAAAGAGAAAAAGAAAGCATCTAAGAACTTGAAAAACT } \\
\text { ACGAATTAGAAAAGAC }\end{array}$ \\
\hline LP2-G-3 & $\begin{array}{l}\text { ATGCTTTCTTTTTCTCTTTTTTACAGATCATCAAGGAAGTAATTATCTAC } \\
\text { TTTTTACAACAAATATAAA }\end{array}$ \\
\hline LP2-G-4(5N) & $\begin{array}{l}\text { GAATAATTCTTCACCTTTAGACATTTTGNNNNNTGTTTTATATTTGTTGT } \\
\text { AAAAAGTAGATAATTACTTC }\end{array}$ \\
\hline LP2-G-4(10N) & $\begin{array}{l}\text { GAATAATTCTTCACCTTTAGACATTTTGNNNNNNNNNNTGTTTTATATT } \\
\text { TGTTGTAAAAAGTAGATAATTACTTC }\end{array}$ \\
\hline LP2-G-4(15N) & $\begin{array}{l}\text { GAATAATTCTTCACCTTTAGACATTTTGNNNNNNNNNNNNNNNTGTTT } \\
\text { TATATTTGTTGTAAAAAGTAGATAATTACTTC }\end{array}$ \\
\hline LP2-G-4(20N) & $\begin{array}{l}\text { GAATAATTCTTCACCTTTAGACATTTTGNNNNNNNNNNNNNNNNNNN } \\
\text { NTGTTTTATATTTGTTGTAAAAAGTAGATAATTACTTC }\end{array}$ \\
\hline LP2-G-4(30N) & $\begin{array}{l}\text { GAATAATTCTTCACCTTTAGACATTTTGNNNNNNNNNNNNNNNNNNN } \\
\text { NNNNNNNNNNNTGTTTTATATTTGTTGTAAAAAGTAGATAATTACTTC }\end{array}$ \\
\hline LP2-G-4(40N) & $\begin{array}{l}\text { GAATAATTCTTCACCTTTAGACATTTTGNNNNNNNNNNNNNNNNNNN } \\
\text { NNNNNNNNNNNNNNNNNNNNNTGTTTTATATTTGTTGTAAAAAGTAG } \\
\text { ATAATTACTTC }\end{array}$ \\
\hline LP2-G-5 & $\begin{array}{l}\text { CAATGTCTAAAGGTGAAGAATTATTCACTGGTGTTGTCCCAATTTTGGT } \\
\text { TGAATTAGATG }\end{array}$ \\
\hline LP2-G-6 & $\begin{array}{l}\text { GGAGACAGAAAATTTGTGACCATTAACATCACCATCTAATTCAACCAA } \\
\text { AATTGGGAC }\end{array}$ \\
\hline LP2-G-7 & $\begin{array}{l}\text { TGGTCACAAATTTTCTGTCTCCGGTGAAGGTGAAGGTGATGCTACTTA } \\
\text { CGGTAAATTG }\end{array}$ \\
\hline LP2-G-8 & AGTAGTACAAATAAATTTTAAGGTCAATTTACCGTAAGTAGCATCAC \\
\hline LP3-G-1 & $\begin{array}{l}\text { AAGGTCCTTTCTTCATAATCAATGCTTTCTTTTACTTAATATTTTACTTG } \\
\text { CATTCAGTGAA }\end{array}$ \\
\hline LP3-G-2 & $\begin{array}{l}\text { TTGCAAGACTAGAGGAATATGTATTAAAATTCACTGAATGCAAGTAAA } \\
\text { ATATTAAGTA }\end{array}$ \\
\hline LP3-G-3 & $\begin{array}{l}\text { TAATACATATTCCTCTAGTCTTGCAAAATCGATTTAGAATCAAGATACC } \\
\text { AGCCTAAAA }\end{array}$ \\
\hline LP3-G-4(5N) & $\begin{array}{l}\text { CACCAGTGAATAATTCTTCACCTTTAGACATTTTGNNNNNTTTTAGGCT } \\
\text { GGTATCTTGATTCTAAA }\end{array}$ \\
\hline LP3-G-4(10N) & $\begin{array}{l}\text { CACCAGTGAATAATTCTTCACCTTTAGACATTTTGNNNNNNNNNNTTTT } \\
\text { AGGCTGGTATCTTGATTCTAAA }\end{array}$ \\
\hline LP3-G-4(15N) & $\begin{array}{l}\text { CACCAGTGAATAATTCTTCACCTTTAGACATTTTGNNNNNNNNNNNNN } \\
\text { NNTTTTAGGCTGGTATCTTGATTCTAAA }\end{array}$ \\
\hline LP3-G-4(20N) & $\begin{array}{l}\text { CACCAGTGAATAATTCTTCACCTTTAGACATTTTGNNNNNNNNNNNNN } \\
\text { NNNNNNNTTTTAGGCTGGTATCTTGATTCTAAA }\end{array}$ \\
\hline LP3-G-4(30N) & $\begin{array}{l}\text { CACCAGTGAATAATTCTTCACCTTTAGACATTTTGNNNNNNNNNNNNN } \\
\text { NNNNNNNNNNNNNNNNNTTTTAGGCTGGTATCTTGATTCTAAA }\end{array}$ \\
\hline LP3-G-4(40N) & $\begin{array}{l}\text { CACCAGTGAATAATTCTTCACCTTTAGACATTTTGNNNNNNNNNNNNN } \\
\text { NNNNNNNNNNNNNNNNNNNNNNNNNNNTTTTAGGCTGGTATCTTGAT }\end{array}$ \\
\hline
\end{tabular}




\begin{tabular}{|c|c|}
\hline & TCTAAA \\
\hline LP3-G-5 & $\begin{array}{l}\text { AAAGGTGAAGAATTATTCACTGGTGTTGTCCCAATTTTGGTTGAATTA } \\
\text { GATGGTGATGT }\end{array}$ \\
\hline LP3-G-6 & $\begin{array}{l}\text { CACCGGAGACAGAAAATTTGTGACCATTAACATCACCATCTAATTCAA } \\
\text { CCAAAAT }\end{array}$ \\
\hline LP3-G-7 & $\begin{array}{l}\text { CACAAATTTTCTGTCTCCGGTGAAGGTGAAGGTGATGCTACTTACGGT } \\
\text { AAATTGAC }\end{array}$ \\
\hline LP3-G-8 & AGTAGTACAAATAAATTTTAAGGTCAATTTACCGTAAGTAGCATC \\
\hline LP4-G-1 & $\begin{array}{l}\text { TTGCAACATTTACTATTTTCCCTTCTTACGTAAATATTTTTCTTTTTAATT } \\
\text { CTAAATCAATCTTTTTC }\end{array}$ \\
\hline LP4-G-2 & $\begin{array}{l}\text { TAAGCAAGAAAAGAATACAAACAAAAAATTGAAAAAGATTGATTTAG } \\
\text { AATTAAAAAGAAAAATA }\end{array}$ \\
\hline LP4-G-3 & $\begin{array}{l}\text { AATTTTTTGTTTGTATTCTTTTCTTGCTTAAATCTATAACTACAAAAAAC } \\
\text { ACATACATAAACTA }\end{array}$ \\
\hline LP4-G-4(5N) & $\begin{array}{l}\text { GTGAATAATTCTTCACCTTTAGACATTTTGNNNNNTTTTAGTTTATGTAT } \\
\text { GTGTTTTTTGTAGTTATA }\end{array}$ \\
\hline LP4-G-4(10N) & $\begin{array}{l}\text { GTGAATAATTCTTCACCTTTAGACATTTTGNNNNNNNNNNTTTTAGTTT } \\
\text { ATGTATGTGTTTTTTGTAGTTATA }\end{array}$ \\
\hline LP4-G-4(15N) & $\begin{array}{l}\text { GTGAATAATTCTTCACCTTTAGACATTTTGNNNNNNNNNNNNNNNTTT } \\
\text { TAGTTTATGTATGTGTTTTTTGTAGTTATA }\end{array}$ \\
\hline LP4-G-4(20N) & $\begin{array}{l}\text { GTGAATAATTCTTCACCTTTAGACATTTTGNNNNNNNNNNNNNNNNNN } \\
\text { NNTTTTAGTTTATGTATGTGTTTTTTGTAGTTATA }\end{array}$ \\
\hline LP4-G-4(30N) & $\begin{array}{l}\text { GTGAATAATTCTTCACCTTTAGACATTTTGNNNNNNNNNNNNNNNNNN } \\
\text { NNNNNNNNNNNNTTTTAGTTTATGTATGTGTTTTTTGTAGTTATA }\end{array}$ \\
\hline LP4-G-4(40N) & $\begin{array}{l}\text { GTGAATAATTCTTCACCTTTAGACATTTTGNNNNNNNNNNNNNNNNNN } \\
\text { NNNNNNNNNNNNNNNNNNNNNNTTTTAGTTTATGTATGTGTTTTTTGT } \\
\text { AGTTATA }\end{array}$ \\
\hline LP4-G-5 & $\begin{array}{l}\text { TGTCTAAAGGTGAAGAATTATTCACTGGTGTTGTCCCAATTTTGGTTGA } \\
\text { ATTAGATGG }\end{array}$ \\
\hline LP4-G-6 & $\begin{array}{l}\text { GGAGACAGAAAATTTGTGACCATTAACATCACCATCTAATTCAACCAA } \\
\text { AATTGGG }\end{array}$ \\
\hline LP4-G-7 & $\begin{array}{l}\text { GGTCACAAATTTTCTGTCTCCGGTGAAGGTGAAGGTGATGCTACTTAC } \\
\text { GGTAAATTG }\end{array}$ \\
\hline LP4-G-8 & AGTAGTACAAATAAATTTTAAGGTCAATTTACCGTAAGTAGCATCAC \\
\hline LP9-G-1 & $\begin{array}{l}\text { CACACAATGAGATTTGTAGTACAGTTCTATTCTCTCTCTTGCATAAATA } \\
\text { AGAAATTCATCAAG }\end{array}$ \\
\hline LP9-G-2 & $\begin{array}{l}\text { TGTGTGTTGGTGAAATATCAAACCAAGTTCTTGATGAATTTCTTATTTAT } \\
\text { GCAAGAGAG }\end{array}$ \\
\hline LP9-G-3 & $\begin{array}{l}\text { TTGGTTTGATATTTCACCAACACACACAAAAAACAGTACTTCACTAAA } \\
\text { TTTACACACAAAAC }\end{array}$ \\
\hline LP9-G-4(5N) & $\begin{array}{l}\text { CAGTGAATAATTCTTCACCTTTAGACATTTTGNNNNNTTTGTTTTGTGT } \\
\text { GTAAATTTAGTGAAGTACTG }\end{array}$ \\
\hline
\end{tabular}




\begin{tabular}{|c|c|}
\hline LP9-G-4(10N) & $\begin{array}{l}\text { CAGTGAATAATTCTTCACCTTTAGACATTTTGNNNNNNNNNNTTTGTTT } \\
\text { TGTGTGTAAATTTAGTGAAGTACTG }\end{array}$ \\
\hline LP9-G-4(15N) & $\begin{array}{l}\text { CAGTGAATAATTCTTCACCTTTAGACATTTTGNNNNNNNNNNNNNNNT } \\
\text { TTGTTTTGTGTGTAAATTTAGTGAAGTACTG }\end{array}$ \\
\hline LP9-G-4(20N) & $\begin{array}{l}\text { CAGTGAATAATTCTTCACCTTTAGACATTTTGNNNNNNNNNNNNNNNN } \\
\text { NNNNTTTGTTTTGTGTGTAAATTTAGTGAAGTACTG }\end{array}$ \\
\hline LP9-G-4(30N) & $\begin{array}{l}\text { CAGTGAATAATTCTTCACCTTTAGACATTTTGNNNNNNNNNNNNNNNN } \\
\text { NNNNNNNNNNNNNNTTTGTTTTGTGTGTAAATTTAGTGAAGTACTG }\end{array}$ \\
\hline LP9-G-4(40N) & $\begin{array}{l}\text { CAGTGAATAATTCTTCACCTTTAGACATTTTGNNNNNNNNNNNNNNNN } \\
\text { NNNNNNNNNNNNNNNNNNNNNNNNTTTGTTTTGTGTGTAAATTTAGT } \\
\text { GAAGTACTG }\end{array}$ \\
\hline LP9-G-5 & $\begin{array}{l}\text { GTCTAAAGGTGAAGAATTATTCACTGGTGTTGTCCCAATTTTGGTTGAA } \\
\text { TTAGATGGTGA }\end{array}$ \\
\hline LP9-G-6 & $\begin{array}{l}\text { CCGGAGACAGAAAATTTGTGACCATTAACATCACCATCTAATTCAACC } \\
\text { AAAATTGG }\end{array}$ \\
\hline LP9-G-7 & $\begin{array}{l}\text { GTCACAAATTTTCTGTCTCCGGTGAAGGTGAAGGTGATGCTACTTACG } \\
\text { GTAAATTGACC }\end{array}$ \\
\hline LP9-G-8 & AGTAGTACAAATAAATTTTAAGGTCAATTTACCGTAAGTAGCATCA \\
\hline LTCV-1-1 & $\begin{array}{l}\text { СТTTCACTCTCCTTGCAATCAGATTTGGGTTTGTTCCCTTTATTTTCATA } \\
\text { TTTCTTGTCATATTC }\end{array}$ \\
\hline LTCv-1-2 & $\begin{array}{l}\text { GAGGTTATGAGTAGAAAATAATAATTGAGAAAGGAATATGACAAGAAA } \\
\text { TATGAAAATAAAGGGAA }\end{array}$ \\
\hline LTCV-1-3 & $\begin{array}{l}\text { TTTCTCAATTATTATTTTCTACTCATAACCTCACGCAAAATAACACAGTC } \\
\text { AAATCAATC }\end{array}$ \\
\hline LTC $v-1-4(5 N)$ & $\begin{array}{l}\text { ACAATGCAGATATCGGAAGAATGTTTCATTTTGNNNNNTTTGATTGATT } \\
\text { TGACTGTGTTATTTTGCG }\end{array}$ \\
\hline LTCv-1-4(20N) & $\begin{array}{l}\text { ACAATGCAGATATCGGAAGAATGTTTCATTTTGNNNNNNNNNNNNNN } \\
\text { NNNNNNTTTGATTGATTTGACTGTGTTATTTTGCG }\end{array}$ \\
\hline LTCv-1-4(40N) & $\begin{array}{l}\text { ACAATGCAGATATCGGAAGAATGTTTCATTTTGNNNNNNNNNNNNNN } \\
\text { NNNNNNNNNNNNNNNNNNNNNNNNNNTTTGATTGATTTGACTGTGTT } \\
\text { ATTTTGCG }\end{array}$ \\
\hline LTCV-1-5 & $\begin{array}{l}\text { AACATTCTTCCGATATCTGCATTGTTGGTGCTGGTATTTCTGGTTTGAC } \\
\text { GTGCG }\end{array}$ \\
\hline LTCV-1-6 & $\begin{array}{l}\text { CGGCATGCCGGGCTGTCCAGCAGATGGCTTGCGCACGTCAAACCAGA } \\
\text { AATAC }\end{array}$ \\
\hline LTCv-1-7 & $\begin{array}{l}\text { TGGACAGCCCGGCATGCCGTGGTCTGAGCCTGCGTATCTTTGACATGC } \\
\text { AGCAAGAAGC }\end{array}$ \\
\hline LTCV-1-8 & TTTGCTGCGGATACGGCCACCGGCTTCTTGCTGCATGTCAAAGATA \\
\hline LTC1-2-1 & $\begin{array}{l}\text { AATTGGTCAATGCAAGAAATACATATTTGGTCTTTTCTAATTCGTAGTTT } \\
\text { TTCAAGTTCT }\end{array}$ \\
\hline LTC1-2-2 & $\begin{array}{l}\text { GATGATCTGTAAAAAAGAGAAAAAGAAAGCATCTAAGAACTTGAAAA } \\
\text { ACTACGAATTAGAA }\end{array}$ \\
\hline
\end{tabular}




\begin{tabular}{|c|c|}
\hline LTC1-2-3 & $\begin{array}{l}\text { ТTCTTTTTCTCTTTTTTACAGATCATCAAGGAAGTAATTATCTACTTTTT } \\
\text { ACAACAAATATAAAAC }\end{array}$ \\
\hline LTC1-2-4(5N) & $\begin{array}{l}\text { GGGAAATCCAGAATGCTCATTTTGNNNNNTGTTTTATATTTGTTGTAAA } \\
\text { AAGTAGATAATTA }\end{array}$ \\
\hline LTC1-2-4(20N) & $\begin{array}{l}\text { GGGAAATCCAGAATGCTCATTTTGNNNNNNNNNNNNNNNNNNNNTGT } \\
\text { TTTATATTTGTTGTAAAAAGTAGATAATTA }\end{array}$ \\
\hline LTC1-2-4(40N) & $\begin{array}{l}\text { GGGAAATCCAGAATGCTCATTTTGNNNNNNNNNNNNNNNNNNNNNN } \\
\text { NNNNNNNNNNNNNNNNNNTGTTTTATATTTGTTGTAAAAAGTAGATAA } \\
\text { TTA }\end{array}$ \\
\hline LTC1-2-5 & $\begin{array}{l}\text { TGAGCATTCTGGATTTCCCGCGTATCCACTTCCGTGGCTGGGCCCGTGT } \\
\text { CAAT }\end{array}$ \\
\hline LTC1-2-6 & $\begin{array}{l}\text { TGTGGCCGTGCGGATCGCGGTTCGCGGTCGGCGCATTGACACGGGCC } \\
\text { CAGCCA }\end{array}$ \\
\hline LTC1-2-7 & $\begin{array}{l}\text { CGCGATCCGCACGGCCACATCGATATGGCCAGCAATACCGTGGCGATG } \\
\text { GCGGG }\end{array}$ \\
\hline LTC1-2-8 & AGGATGGCGTGCCAGGTCGAACGGCTCACCCGCCATCGCCACGGTAT \\
\hline LTC2-3-1 & $\begin{array}{l}\text { AAAAGGTCCTTTCTTCATAATCAATGCTTTCTTTTACTTAATATTTTACT } \\
\text { TGCATTCAGTGAA }\end{array}$ \\
\hline LTC2-3-2 & $\begin{array}{l}\text { TGCAAGACTAGAGGAATATGTATTAAAATTCACTGAATGCAAGTAAAA } \\
\text { TATTAAGTAAA }\end{array}$ \\
\hline LTC2-3-3 & $\begin{array}{l}\text { TTTAATACATATTCCTCTAGTCTTGCAAAATCGATTTAGAATCAAGATAC } \\
\text { CAGCCTAAAA }\end{array}$ \\
\hline LTC2-3-4(5N) & $\begin{array}{l}\text { ACAGTGGTGGCTCACGGTTCTCCATTTTGNNNNNTTTTAGGCTGGTAT } \\
\text { CTTGATTCTAAAT }\end{array}$ \\
\hline LTC2-3-4(20N) & $\begin{array}{l}\text { ACAGTGGTGGCTCACGGTTCTCCATTTTGNNNNNNNNNNNNNNNNNN } \\
\text { NNTTTTAGGCTGGTATCTTGATTCTAAAT }\end{array}$ \\
\hline LTC2-3-4(40N) & $\begin{array}{l}\text { ACAGTGGTGGCTCACGGTTCTCCATTTTGNNNNNNNNNNNNNNNNNN } \\
\text { NNNNNNNNNNNNNNNNNNNNNNTTTTAGGCTGGTATCTTGATTCTAA } \\
\text { AT }\end{array}$ \\
\hline LTC2-3-5 & $\begin{array}{l}\text { AACCGTGAGCCACCACTGTTGCCAGCCCGTTGGAGCAGCGCCTATGT } \\
\text { CTCTT }\end{array}$ \\
\hline LTC2-3-6 & $\begin{array}{l}\text { TCAGCTGGTCATCCGGCAGCATCGGGCTCCAATAAGAGACATAGGCGC } \\
\text { TGCT }\end{array}$ \\
\hline LTC2-3-7 & $\begin{array}{l}\text { CTGCCGGATGACCAGCTGACCAGCGGCTATTGCTGGTTCGACTATGAA } \\
\text { CGTGAC }\end{array}$ \\
\hline LTC2-3-8 & GAACAGGCCGTCAATACGACAGATGTCACGTTCATAGTCGAACCA \\
\hline LTC3-4-1 & $\begin{array}{l}\text { TTGCAACATTTACTATTTTCCCTTCTTACGTAAATATTTTTCTTTTTAATT } \\
\text { CTAAATCAATCTTTTTC }\end{array}$ \\
\hline LTC3-4-2 & $\begin{array}{l}\text { AAGCAAGAAAAGAATACAAACAAAAAATTGAAAAAGATTGATTTAGA } \\
\text { ATTAAAAAGAAAAATA }\end{array}$ \\
\hline LTC3-4-3 & $\begin{array}{l}\text { AАTTTTTTGTTTGTATTCTTTTCTTGCTTAAATCTATAACTACAAAAAAC } \\
\text { ACATACATAAACT }\end{array}$ \\
\hline
\end{tabular}




\begin{tabular}{|c|c|}
\hline LTC3-4-4(5N) & $\begin{array}{l}\text { CACCAATGACCAGAATCTTCATTTTGNNNNNTTTTAGTTTATGTATGTG } \\
\text { TTTTTTGTAGTTATA }\end{array}$ \\
\hline LTC3-4-4(20N) & $\begin{array}{l}\text { CACCAATGACCAGAATCTTCATTTTGNNNNNNNNNNNNNNNNNNNNT } \\
\text { TTTAGTTTATGTATGTGTTTTTTGTAGTTATA }\end{array}$ \\
\hline LTC3-4-4(40N) & $\begin{array}{l}\text { CACCAATGACCAGAATCTTCATTTTGNNNNNNNNNNNNNNNNNNNNN } \\
\text { NNNNNNNNNNNNNNNNNNNTTTTAGTTTATGTATGTGTTTTTTGTAGT } \\
\text { TATA }\end{array}$ \\
\hline LTC3-4-5 & $\begin{array}{l}\text { TGAAGATTCTGGTCATTGGTGCTGGTCCAGCTGGTCTGGTTTTCGCAT } \\
\text { CCCAA }\end{array}$ \\
\hline LTC3-4-6 & $\begin{array}{l}\text { TGTCAATGGCCCACAAAGGGCGTGCCTGCTTCAGTTGGGATGCGAAA } \\
\text { ACCAGA }\end{array}$ \\
\hline LTC3-4-7 & $\begin{array}{l}\text { CCTTTGTGGGCCATTGACATCGTGGAGAAGAATGACGAGCAAGAAGT } \\
\text { GCTGGG }\end{array}$ \\
\hline LTC3-4-8 & CGGACGGCCAGGCAGCACGACACCCCAGCCCAGCACTTCTTGCTCGT \\
\hline LTC4-5-1 & $\begin{array}{l}\text { CACACAATGAGATTTGTAGTACAGTTCTATTCTCTCTCTTGCATAAATA } \\
\text { AGAAATTCATCAAG }\end{array}$ \\
\hline LTC4-5-2 & $\begin{array}{l}\text { GTGTGTGTTGGTGAAATATCAAACCAAGTTCTTGATGAATTTCTTATTT } \\
\text { ATGCAAGAGAG }\end{array}$ \\
\hline LTC4-5-3 & $\begin{array}{l}\text { TTGGTTTGATATTTCACCAACACACACAAAAAACAGTACTTCACTAAA } \\
\text { TTTACACACAAAAC }\end{array}$ \\
\hline LTC4-5-4(5N) & $\begin{array}{l}\text { CACCAACGATAATCGCACGTTTCATTTTGNNNNNTTTGTTTTGTGTGTA } \\
\text { AATTTAGTGAAGTACTG }\end{array}$ \\
\hline LTC4-5-4(20N) & $\begin{array}{l}\text { CACCAACGATAATCGCACGTTTCATTTTGNNNNNNNNNNNNNNNNNN } \\
\text { NNTTTGTTTTGTGTGTAAATTTAGTGAAGTACTG }\end{array}$ \\
\hline LTC4-5-4(40N) & $\begin{array}{l}\text { CACCAACGATAATCGCACGTTTCATTTTGNNNNNNNNNNNNNNNNNN } \\
\text { NNNNNNNNNNNNNNNNNNNNNNTTTGTTTTGTGTGTAAATTTAGTGA } \\
\text { AGTACTG }\end{array}$ \\
\hline LTC4-5-5 & $\begin{array}{l}\text { GAAACGTGCGATTATCGTTGGTGGCGGCCTGGCGGGTGGCCTGACCG } \\
\text { CGATCT }\end{array}$ \\
\hline LTC4-5-6 & $\begin{array}{l}\text { ACGACGTGCACTTCGTAGCCACGCTTCGCCAGGTAGATCGCGGTCAG } \\
\text { GCCACC }\end{array}$ \\
\hline LTC4-5-7 & $\begin{array}{l}\text { GCTACGAAGTGCACGTCGTGGAGAAGCGTGGTGATCCTCTGCGCGAT } \\
\text { CTGAGC }\end{array}$ \\
\hline LTC4-5-8 & ACGGCTGCTAACAACGTCCACGTAAGAGCTCAGATCGCGCAGAGGA \\
\hline
\end{tabular}

Table S2. Summarization of the sequences of bases of N (A/T/C/G) introduced by regulatory linkers. Some groups of de novo synthetic linkers were ligated into pEASY-Blunt, transformed into E. coli, and extracted for plasmid sequencing. For each group, the sequencing results of 20 picked colonies were summarized in the following Table. An example of abbreviation in the table was LP1-G(5N), which meant the linker between $\mathrm{P} 1$ and $g f p$ 's ORF where 5 bases of $\mathrm{N}$ were introduced. 
130 Another example was LP1-A(20N) representative of the linker between P1 and vioA

131 where 20 bases of $\mathrm{N}$ were introduced.

\begin{tabular}{|c|c|c|c|}
\hline $\begin{array}{l}\text { Linker's } \\
\text { name }\end{array}$ & No. & 5' to 3'Sequence (... NNN...CAAAATG...) & $\underline{\mathrm{GC}} \%$ \\
\hline \multirow[t]{20}{*}{ LP1-G(5N) } & 1 & ...CACGGCAAAATG... & $80 \%$ \\
\hline & 2 & ...AGGTACAAAATG... & $40 \%$ \\
\hline & 3 & ...CAGGCCAAAATG... & $80 \%$ \\
\hline & 4 & ...TGCATCAAATG... & $40 \%$ \\
\hline & 5 & ...TGGTACAAAATG... & $40 \%$ \\
\hline & 6 & ...AGGTCCAAAATG... & $60 \%$ \\
\hline & 7 & ...GGGTTCAAAATG... & $60 \%$ \\
\hline & 8 & ...TTGGGCAAAATG... & $60 \%$ \\
\hline & 9 & ... & $60 \%$ \\
\hline & 10 & ...GCACTCAAAATG... & $60 \%$ \\
\hline & 11 & ... & $80 \%$ \\
\hline & 12 & ...GGCTGCAAAATG... & $80 \%$ \\
\hline & 13 & ...CATAGCAAAATG... & $40 \%$ \\
\hline & 14 & ... TGGTACAAAATG... & $40 \%$ \\
\hline & 15 & ...GGCGACAAAATG... & $80 \%$ \\
\hline & 16 & ... & $60 \%$ \\
\hline & 17 & ... & $20 \%$ \\
\hline & 18 & ...TAGTACAAAATG... & $20 \%$ \\
\hline & 19 & ...TTGGACAAAATG... & $40 \%$ \\
\hline & 20 & ... TTAACCAAAATG... & $20 \%$ \\
\hline \multirow[t]{20}{*}{ LP1-G(20N) } & 1 & .... AACCAACCGCAAGGCAATGTCAAAATG... & $50 \%$ \\
\hline & 2 & ...ATACGGGCGAGAGATACAACCAAAATG... & $50 \%$ \\
\hline & 3 & ....AAGAGCAAAGCAAGAGCGTTCAAAATG... & $45 \%$ \\
\hline & 4 & ...GCTTATTAATGACAACCGAACAAAATG... & $35 \%$ \\
\hline & 5 & ...TATACGGACCAACAAAAGAACAAAATG... & $35 \%$ \\
\hline & 6 & ...CCTACATGCAACCTGCCAAGCAAAATG... & $55 \%$ \\
\hline & 7 & ...TGATCAGCCATCTCACACAACAAAATG... & $45 \%$ \\
\hline & 8 & ...CGATGGCAGGTCCATAGAGACAAAATG... & $55 \%$ \\
\hline & 9 & ...CTTATTGGTAAAAACAAGATCAAAATG... & $25 \%$ \\
\hline & 10 & ...TCGGGCACTAAAGACAGGACCAAAATG... & $55 \%$ \\
\hline & 11 & ...TACTTAACGCCCGCCCGAGGCAAAATG... & $65 \%$ \\
\hline & 12 & ...GGCACAGGCTTCAGGTAAGTCAAAATG... & $55 \%$ \\
\hline & 13 & ...GACACCAGTCGGAAAGACACCAAAATG... & $55 \%$ \\
\hline & 14 & ....AACCTTCGTAGTAAAGGAATCAAAATG... & $35 \%$ \\
\hline & 15 & ....AGGGCGGCAATCGGCTAAACCAAAATG... & $60 \%$ \\
\hline & 16 & ...GAGCGCAGGGCCACTGTGAACAAAATG... & $65 \%$ \\
\hline & 17 & ...ATGTGCCAGCGGAACTAATCCAAAATG... & $50 \%$ \\
\hline & 18 & .... ACTATCCAAGGCCCATAAGACAAAATG... & $45 \%$ \\
\hline & 19 & .... TGCCTCATAGCCCGCAACATCAAAATG... & $55 \%$ \\
\hline & 20 & .... СТACCCATGTACTAACACAACAAAATG... & $40 \%$ \\
\hline
\end{tabular}




\begin{tabular}{|c|c|c|c|}
\hline \multirow[t]{40}{*}{ LP1-G(40N) } & \multirow[t]{2}{*}{1} & .... TGAGCGGCCCTTTAGGCCTCGCCACGGCTTAGTGCCA & $65 \%$ \\
\hline & & СТCCAAAATG... & \\
\hline & \multirow[t]{2}{*}{2} & .... СTCCCTACTATCAAATGACATGTTGCAACCGGGATTCA & $45 \%$ \\
\hline & & $\underline{\text { CTCAAAATG... }}$ & \\
\hline & \multirow[t]{2}{*}{3} & ...GTCCGTGGCATCCAGAGAGATACTACTTAAGTTGCTAA & $47.5 \%$ \\
\hline & & GCCAAAATG... & \\
\hline & \multirow[t]{2}{*}{4} & ...GTGGTCGCACCGGCTATCAGTATCAACAGACGCCCGC & $62.5 \%$ \\
\hline & & СCTCAAAATG... & \\
\hline & \multirow[t]{2}{*}{5} & ...CCCCAATGAAAAAGTTGGAAAAAGAAAGCTACGCGA & $40 \%$ \\
\hline & & GATTCAAAATG... & \\
\hline & \multirow[t]{2}{*}{6} & ...GAGAGAAGTGTCAACGTATGATTGAAGGGTTCGATTA & $42.5 \%$ \\
\hline & & CTGCAAAATG... & \\
\hline & \multirow[t]{2}{*}{7} & ...GCTGAACCTATGAGTGATGCTACTTACGGTAAATTGATT & $37.5 \%$ \\
\hline & & 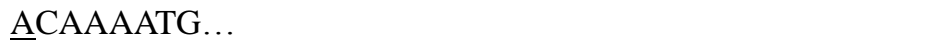 & \\
\hline & \multirow[t]{2}{*}{8} & ...CGACTTCCAAATTACCAACTCGCTTACTACCAATAACA & $37.5 \%$ \\
\hline & & $\underline{\text { TTCAAAATG... }}$ & \\
\hline & \multirow[t]{2}{*}{9} & .... TCTAAAACCGGGAATCTTTTCCTAGACTGAGACTTGCC & $45 \%$ \\
\hline & & $\underline{\text { TGCAAAATG... }}$ & \\
\hline & \multirow[t]{2}{*}{10} & ...TGTTCCCCAACTATATTGGACTCACTCTGGAGGTAGAT & $45 \%$ \\
\hline & & GACAAAATG... & \\
\hline & \multirow[t]{2}{*}{11} & .... TGTTCCCCAACTATATTGGACTCACTCTGGAGGTAGAT & $45 \%$ \\
\hline & & $\underline{\text { GACAAAATG... }}$ & \\
\hline & \multirow[t]{2}{*}{12} & ....CCTCGTGCGTAGTCCGCTCAAAGGAATGGCGCGATCA & $57.5 \%$ \\
\hline & & CAACAAAATG... & \\
\hline & \multirow[t]{2}{*}{13} & ...TCAACCTAAGCGCATAAGCACCTAAGGAACTACGTGC & $47.5 \%$ \\
\hline & & TCACAAAATG... & \\
\hline & \multirow[t]{2}{*}{14} & ....AAGGAATTTTCCTCTTGCTCGCGTCCTGCCCAAAAGGT & $50 \%$ \\
\hline & & $\underline{\text { GTCAAAATG... }}$ & \\
\hline & \multirow[t]{2}{*}{15} & ...CAGACTCATATTCACATTCCCCTCGAAACAAATAAGGG & $40 \%$ \\
\hline & & $\underline{\text { ATCAAAATG... }}$ & \\
\hline & \multirow[t]{2}{*}{16} & ...GACCCCTCAGCGCCGTACTCAGACTATAATTTGCACGA & $52.5 \%$ \\
\hline & & $\underline{\text { ACCAAAATG... }}$ & \\
\hline & \multirow[t]{2}{*}{17} & .... AGTATATCCTAGAAACGGACACGCTGCTCGGTGAAAA & $50 \%$ \\
\hline & & $\underline{\text { CCGCAAAATG... }}$ & \\
\hline & \multirow[t]{2}{*}{18} & .... ACCAATAAAATTGATAAATCCAAATTCTCTGCCATAAAT & $27.5 \%$ \\
\hline & & CCAAAATG... & \\
\hline & \multirow[t]{2}{*}{19} & .... AGTGCAATAGGACGTTACATTTCATTCACCCTCCCCTG & $47.5 \%$ \\
\hline & & $\underline{\text { AGCAAAATG } \ldots}$ & \\
\hline & \multirow[t]{2}{*}{20} & .... ACACTTAGATTCTCCACCTCCCCCAGAAGCAGACCGG & $57.5 \%$ \\
\hline & & GACCAAAATG... & \\
\hline \multirow[t]{4}{*}{ LP1-A(5N) } & 1 & ...CAGACCAAAATG... & $60 \%$ \\
\hline & 2 & ...CTTCACAAAATG... & $40 \%$ \\
\hline & 3 & ...TGCGCCAAAATG... & $80 \%$ \\
\hline & 4 & ...CGCGTCAAAATG... & $80 \%$ \\
\hline
\end{tabular}




\begin{tabular}{|c|c|c|c|}
\hline & 5 & ...TGGATCAAAATG... & $40 \%$ \\
\hline & 6 & ...CCAAGCAAAATG... & $60 \%$ \\
\hline & 7 & .... ATCCGCAAAATG... & $60 \%$ \\
\hline & 8 & ...TCCATCAAAATG... & $40 \%$ \\
\hline & 9 & ...GTGTGCAAAATG... & $60 \%$ \\
\hline & 10 & ...ATTGGCAAAATG... & $40 \%$ \\
\hline & 11 & ...TGCGCCAAAATG... & $80 \%$ \\
\hline & 12 & .... & $20 \%$ \\
\hline & 13 & ...CGCCCCAAAATG ... & $100 \%$ \\
\hline & 14 & ...CGTTCCAAAATG... & $60 \%$ \\
\hline & 15 & ...CGCGCCAAAATG... & $100 \%$ \\
\hline & 16 & ...ACTGCCAAAATG... & $80 \%$ \\
\hline & 17 & ...CTGACCAAAATG... & $60 \%$ \\
\hline & 18 & ...TACGGCAAAATG... & $60 \%$ \\
\hline & 19 & ...CGTCGCAAAATG... & $80 \%$ \\
\hline & 20 & ...CTCGACAAAATG... & $60 \%$ \\
\hline LP1-A(20N) & 1 & ....CCCCGGCGGCGCTAGCCCATCAAAATG... & $80 \%$ \\
\hline & 2 & ...CTTCCGCCCGTAGTAGCATTCAAAATG... & $55 \%$ \\
\hline & 3 & ...GGACGGGGACGCGCTCCGCTCAAAATG... & $80 \%$ \\
\hline & 4 & ...TTCCCTTTACCAAGGGCAGCCAAAATG... & $55 \%$ \\
\hline & 5 & .... CACATTAGCAGCCCGGCTGGCAAAATG... & $65 \%$ \\
\hline & 6 & ...TGTCGGCCCCATTAATAGGGCAAAATG... & $55 \%$ \\
\hline & 7 & ...CCCGGCCGGCACCACAGCTGCAAAATG... & $80 \%$ \\
\hline & 8 & ....CGCTGCTCGGTCTGAACCACCAAAATG... & $65 \%$ \\
\hline & 9 & ...GCTGACCAAATCGCACTTTGCAAAATG... & $50 \%$ \\
\hline & 10 & ...GATCCGTTCGGCTTAGTGCCCAAAATG... & $60 \%$ \\
\hline & 11 & ...CCGTGCACAGAGTATACCACCAAAATG... & $55 \%$ \\
\hline & 12 & ...GCGCTGGCTGCGTGGCACCGCAAAATG... & $80 \%$ \\
\hline & 13 & ....CCCCAGACCGCTTGTAATGCAAAATG... & $60 \%$ \\
\hline & 14 & ...GCGCTGGCTGCGTGGCACCGCAAAATG... & $80 \%$ \\
\hline & 15 & .... ATCCAAGGGGTTTTCCACCCCAAAATG... & $55 \%$ \\
\hline & 16 & ...GAGGTAAGCAGGAATTCTCACAAAATG... & $45 \%$ \\
\hline & 17 & ...ACTGTAGGTTCCGGAGGAGGCAAAATG... & $60 \%$ \\
\hline & 18 & ...TGCGAGGGGTGCAGTAAGGGCAAAATG... & $65 \%$ \\
\hline & 19 & ...CACTTGGCCGCATGAGCAGGCAAAATG... & $65 \%$ \\
\hline & 20 & ...CACATTAGCAGCCCGGCTGGCAAAATG... & $65 \%$ \\
\hline LP1-A(40N) & 1 & ...GCGAACTGTGACTGATGCACGGTCAACTCAACGTCCA & $52.5 \%$ \\
\hline & & $\underline{\text { ATGCAAAATG } \ldots}$ & \\
\hline & 2 & $\begin{array}{l}\text {......CCCTGCCAGCCCCCATACCCGGCGACGAATAACTGC } \\
\text { CAACCAAAATG... }\end{array}$ & $65 \%$ \\
\hline & 3 & ...CGCGCCGACATAGCCTTTACAACACAATACGTCGTAAT & $45 \%$ \\
\hline & & $\underline{\text { AACAAAATG } \ldots}$ & \\
\hline & 4 & $\begin{array}{l}\text {...CCGCCGCTCCAGTCGCGAGTCACTGAAGACACGGACG } \\
\text { TGCCAAAATG... }\end{array}$ & $67.5 \%$ \\
\hline
\end{tabular}




\begin{tabular}{|c|c|c|}
\hline \multirow[t]{2}{*}{5} & .GCGCGCATTCCCTTGTGTCGTTCCAAGCCTACGCCCGG & $67.5 \%$ \\
\hline & $\underline{\text { GGCAAAATG... }}$ & \\
\hline \multirow[t]{2}{*}{6} & ...GCGAGACGGGCACGGGGAAACCGCGTTTGGTTACCTA & $65 \%$ \\
\hline & $\underline{\text { GGGCAAAATG... }}$ & \\
\hline \multirow[t]{2}{*}{7} & ...CACCCGCAGCCCGCGAGGAAGACTGCAAATCTTTGCA & $57.5 \%$ \\
\hline & CATCAAAATG... & \\
\hline \multirow[t]{2}{*}{8} & ...TCCCCGGCCGAAGCACCATCACCCCTCCTGACAACCG & $65 \%$ \\
\hline & TAGCAAAATG... & \\
\hline \multirow[t]{2}{*}{9} & ....TACTGTGGAACAGATCATTAAAATGGCTCAGAACAATA & $35 \%$ \\
\hline & $\underline{\text { CACAAAATG... }}$ & \\
\hline \multirow[t]{2}{*}{10} & ....GGCACTATTGTAACAGCCCACCGGAATAACAGCGACT & $50 \%$ \\
\hline & $\underline{\text { AAGCAAAATG } \ldots}$ & \\
\hline \multirow[t]{2}{*}{11} & ...GGGCCGGCCGCCCGCATTCCGAGCCCACCAATGGTTC & $72.5 \%$ \\
\hline & CCTCAAAATG... & \\
\hline \multirow[t]{2}{*}{12} & ...CAGCAGGCGCGCCAAAGCGATACCCCAGCGGAACTGC & $62.5 \%$ \\
\hline & TTTCAAAATG... & \\
\hline \multirow[t]{2}{*}{13} & .... TGTGCGGCCGCGTGTCCAGGAACACATGTCCATACCC & $62.5 \%$ \\
\hline & 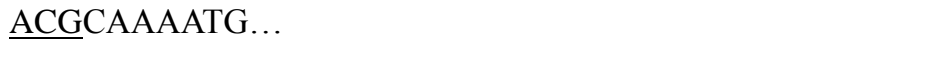 & \\
\hline \multirow[t]{2}{*}{14} & ...CCCTACCCCAAGGCCGCCACCCGGTCCTGCCAAACTAT & $62.5 \%$ \\
\hline & $\underline{\text { ATCAAAATG } \ldots}$ & \\
\hline \multirow[t]{2}{*}{15} & ....CTCCCGAAGTTGCATATTGGGAATGAAAGTATATATAA & $35 \%$ \\
\hline & TCAAAATG... & \\
\hline \multirow[t]{2}{*}{16} & ...CTACCGCCGCCAGGGCGCTGTCGGAAGCCCCATTCAA & $65 \%$ \\
\hline & AGTCAAAATG... & \\
\hline \multirow[t]{2}{*}{17} & ...TGACCCTAGCTTACGGAATTGTGAACGAGGTCAGTGT & $47.5 \%$ \\
\hline & $\underline{\text { ACACAAAATG } \ldots}$ & \\
\hline \multirow[t]{2}{*}{18} & .... CGGCAGCCCACCACAAGTACCACAAACTTGCAGCCCC & $60 \%$ \\
\hline & 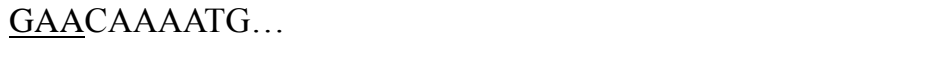 & \\
\hline \multirow[t]{2}{*}{19} & .... ACGGGCGGGGGGCATAAACGTTAACATCGAGGACACC & $60 \%$ \\
\hline & $\underline{\text { CAGCAAAATG... }}$ & \\
\hline \multirow[t]{2}{*}{20} & ...TACGCCCGTTACACACTTAGGACCGTCGCGCAGACAA & $55 \%$ \\
\hline & $\underline{\text { GATCAAAATG... }}$ & \\
\hline
\end{tabular}

Table S3. The raw transcriptional data detected in the qualitative real-time PCR.All of the data were detected by using their each primer. All of the data of the five genes vioA,vioB, vioE, vioD and vioC in each sample strain were normalized by thereference gene $\operatorname{alg} 9$. The values shown here represented the relative strengths of transcription where $5 \mathrm{~N}-43$ was set as the control.

\begin{tabular}{llllll}
\hline Sample & vioA & vioB & vioE & vioD & vioC \\
\hline $5 \mathrm{~N}-12$ & $0.1365 \pm$ & $0.3311 \pm$ & $0.2784 \pm$ & none & none \\
& 0.0106 & 0.0237 & 0.0233 & & \\
$5 \mathrm{~N}-16$ & $0.1002 \pm$ & $1.0053 \pm$ & $0.5982 \pm$ & $0.5990 \pm$ & $0.9543 \pm$ \\
& 0.0089 & 0.0902 & 0.0573 & 0.0763 & 0.0832 \\
\hline
\end{tabular}




\begin{tabular}{llllll}
\hline $5 \mathrm{~N}-17$ & $0.6253 \pm$ & $1.0444 \pm$ & $1.1530 \pm$ & $0.7774 \pm$ & $0.9067 \pm$ \\
& 0.0322 & 0.0573 & 0.0900 & 0.0835 & 0.0963 \\
$5 \mathrm{~N}-25$ & $0.2546 \pm$ & $0.4961 \pm$ & $0.4409 \pm$ & $0.3340 \pm$ & $0.4648 \pm$ \\
& 0.0091 & 0.0231 & 0.033 & 0.0085 & 0.0267 \\
$5 \mathrm{~N}-35$ & $0.7503 \pm$ & $0.8018 \pm$ & $0.6706 \pm$ & $1.1715 \pm$ & $0.8678 \pm$ \\
& 0.0691 & 0.0700 & 0.0421 & 0.1640 & 0.1073 \\
$5 \mathrm{~N}-43$ & $1.0000 \pm$ & $1.0000 \pm$ & $1.0000 \pm$ & $1.0000 \pm$ & $1.0000 \pm$ \\
& 0.0697 & 0.0525 & 0.0351 & 0.0656 & 0.0409 \\
$20 \mathrm{~N}-17$ & $0.2551 \pm$ & $0.4053 \pm$ & $0.4223 \pm$ & $0.9537 \pm$ & $1.1855 \pm$ \\
& 0.0105 & 0.0103 & 0.0155 & 0.0597 & 0.1221 \\
$20 \mathrm{~N}-29$ & $1.4448 \pm$ & $1.6074 \pm$ & $1.0810 \pm$ & $1.2712 \pm$ & $1.1093 \pm$ \\
& 0.0814 & 0.0461 & 0.0350 & 0.2259 & 0.0579 \\
$20 \mathrm{~N}-48$ & $0.7472 \pm$ & $0.9929 \pm$ & $0.4101 \pm$ & $0.9098 \pm$ & $1.4153 \pm$ \\
& 0.0606 & 0.0438 & 0.0285 & 0.1078 & 0.0825 \\
$40 \mathrm{~N}-48$ & $0.5905 \pm$ & $0.3111 \pm$ & $0.1975 \pm$ & $1.2847 \pm$ & $1.0363 \pm$ \\
& 0.0395 & 0.0115 & 0.0062 & 0.0707 & 0.0746 \\
\hline
\end{tabular}

Table S4. The primers used in the qualitative real-time PCR.

\begin{tabular}{ll}
\hline Primer & 5' to 3' Sequence \\
\hline vioA-qF & CCGTGGTGGTTGGACTAC \\
vioA-qR & TTCGCCATCTCGCTATCG \\
vioB-qF & CTGCTGCTGCGTGATGAG \\
vioB-qR & TACCGTGATTCGTCCAATAGTC \\
vio $E$-qF & CTGACCAGCGGCTATTGC \\
vio $E-\mathrm{qR}$ & GACATCCACAGGCGATAACC \\
vio $D-\mathrm{qF}$ & CGAATGTAGCGAAGAGAC \\
vioD-qR & CAGCAGAACCAACTTACC \\
vioC-qF & GACGACCACCGACGAACC \\
vioC-qR & CCCAGCAACAGCACATTACC \\
$a l g 9-\mathrm{qF}$ & CAGGCAATGTCACGGATAG \\
alg $9-\mathrm{qR}$ & GCAGCAGGAAAGAACTTGG \\
\hline
\end{tabular}

